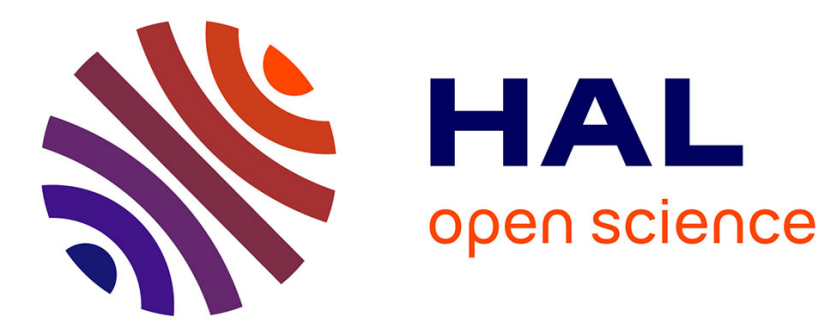

\title{
Evolution of methane density during melting in nanopores
}

E Dundar, C Wexler, L Firlej, P Llewellin, B Kuchta

\section{To cite this version:}

E Dundar, C Wexler, L Firlej, P Llewellin, B Kuchta. Evolution of methane density during melting in nanopores. Journal of Molecular Modeling, 2017, 23 (2), pp.44. 10.1007/s00894-017-3211-5 . hal01813863

\section{HAL Id: hal-01813863 \\ https://hal-amu.archives-ouvertes.fr/hal-01813863}

Submitted on 12 Jun 2018

HAL is a multi-disciplinary open access archive for the deposit and dissemination of scientific research documents, whether they are published or not. The documents may come from teaching and research institutions in France or abroad, or from public or private research centers.
L'archive ouverte pluridisciplinaire HAL, est destinée au dépôt et à la diffusion de documents scientifiques de niveau recherche, publiés ou non, émanant des établissements d'enseignement et de recherche français ou étrangers, des laboratoires publics ou privés. 


\title{
Evolution of methane density during melting in nanopores
}

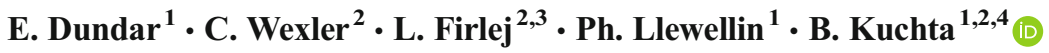

Received: 14 October 2016 / Accepted: 9 January 2017 / Published online: 2 February 2017

(C) Springer-Verlag Berlin Heidelberg 2017

\begin{abstract}
Phase properties of gases adsorbed in small nanopores are mainly determined by the pore size and shape as well as the structural heterogeneity of the adsorbate. Here we analyze the evolution of the melting mechanism that occurs in pores $<3 \mathrm{~nm}$ in size. Melting in slit-shaped graphene pores is compared with melting in SURMOF channel pores with square cross-sections. We show how the melting transformation is related to the adsorption mechanism. We use a graphical representation of the evolution of molecular density as a function of temperature in the nanopores.
\end{abstract}

Keywords Nanopores $\cdot$ Methane $\cdot$ Melting $\cdot$ Structural heterogeneity

\section{Introduction}

Melting and freezing in confined systems are important in many natural processes (e.g., frost heaving and the distribu-

This paper belongs to Topical Collection 7th Conference on Modeling \& Design of Molecular Materials in Trzebnica (MDMM 2016)

B. Kuchta

bogdan.kuchta@univ-amu.fr

1 Aix Marseille University, CNRS, MADIREL (UMR 7246), 13397 Marseille, France

2 Department of Physics and Astronomy, University of Missouri, Columbia, MO 65211, USA

3 Laboratoire Charles Coulomb (L2C), UMR 5221, Université de Montpellier, Montpellier, France

4 Department of Chemistry, Wroclaw University of Technology, 50-370 Wroclaw, Poland tion of liquids in soils and rocks) as well as in lubrication, adhesion, and nanotribology. It is well known that the structures and phase behaviors of confined fluids are quite distinct from those of their bulk counterparts. This is related to the high surface-to-volume ratios that are typical of nanosystems: as the surface atoms are weakly bonded, nanoscale systems usually transform at lower temperatures than their bulk analogs. Experimental methods of determining changes at freezing point in confined systems typically make use of the Gibbs-Thomson equation, which includes the enthalpy of fusion, the pore size, and the corresponding surface tension. However, this approach fails for nanosized pores, whereas numerical simulation remains an effective tool that allows us to circumvent the limitations of bulk models.

A deep understanding of the fundamental mechanisms of phase changes at the nanoscale is crucial to exploring the new field of nanothermodynamics, which considers finite-size effects, nanodimensionality, and extended surface forces. The reduced size of the confined space weakens the intermolecular forces present, so we can expect significant shifts in the transition temperatures. In some cases, we may even observe new surface- or confinement-induced phases. The consequences of this redefinition of properties for nanoscale systems are of theoretical as well as practical interest.

The structural changes that occur in confined geometries have been studied ever since computer modeling became possible [1-3]. Melting in the simplest geometry, slit pores, was analyzed in a paper by Miyahara and Gubbins [1]. There have also been a few reports of solid-solid (hexagonal-tetragonal) transformations in this geometry $[4,5]$. In all cases, the goal of the study was to understand the relation between the mechanism of transformation and (i) the pore size and shape as well as (ii) the strength of the adsorbate-adsorbent interaction [6-8]. However, due to the nanoscale of the systems considered, they are heterogeneous, and the distribution of the 
interactions makes analyzing the mechanism of phase changes under nanoconfinement difficult [9].

Here, we analyze the melting mechanism from the point of view of system heterogeneity as well as its influence on the melting temperature. We use locally defined structural order parameters to follow the space distribution of structural changes in the fluid system as a function of temperature. We base our conclusions on an analysis of a graphical representation of the evolution of fluid density and structure during the melting transformation. We emphasize that qualitatively new information can be obtained from such a representation. This study is an extension of previously published results [9].

\section{Numerical methodology}

The computational approach used was based on GCMC simulations implemented in a homemade software package that was extensively tested and validated previously [10-13]. The carbon slit-shaped framework and the SURMOF structure were considered to be rigid during simulations. All interatomic interactions were modeled using the Lennard-Jones (6-12) potential. The $\mathrm{CH}_{4}$ molecules were considered superatoms (united atom model). The $\mathrm{CH}_{4}-\mathrm{CH}_{4}$ Lennard-Jones parameters were $\sigma_{\mathrm{CH} 4}=3.730 \AA$ and $\varepsilon_{\mathrm{CH} 4}=148.0 \mathrm{~K}$. For methanewall interactions in the slit pores, the Steele 10-4-3 potentials were employed $[14,15]$. Parameters describing fluid-solid interactions were obtained using the Lorentz-Berthelot mixing rules. In the SURMOF structure, all interatomic interactions were modeled using the Lennard-Jones (6-12) potential. The force field model employed here was used and tested in a previous paper [9]. The interactions were cut off at $15 \AA$ and periodic boundary conditions were used. Isotherm points were calculated by averaging the number of adsorbed molecules per unit cell of the material for a given chemical potential $\mu(P)$ and temperature $T$. The methane was modeled as an ideal gas, so, technically, the pressure parameter $P$ is actually a fugacity.

The following order parameter plays the most important role in the analysis presented here:

$\Phi_{n j k}=\left|\left\langle\frac{l}{N_{\mathrm{b}}} \sum_{k=1}^{N_{\mathrm{b}}} \mathrm{e}^{i n \theta_{k}}\right\rangle\right|$

This parameter is defined and calculated for each layer separately. The indices $j$ and $k$ indicate the local character of the order parameter calculation (atom $k$ in layer $j$ ). $\Phi_{n}$ tracks the local structural symmetry of the center-of-mass lattice positions by measuring the average bond order within a plane layer. The centers of mass of each nearest-neighbor pair are connected by a vector ("bond") that has a particular azimuthal orientation $\theta_{k}$. The index $k$ runs over the total number of nearest-neighbor bonds $N_{\mathrm{b}}$ in the adsorbed layer. The order parameter is equal to 1 if the local structure has hexagonal
( $n=6)$ or tetragonal $(n=4)$ solid order, and it is equal to 0 if the structure is a disordered liquid.

The pore structures are shown in Fig. 1. The slit pores of carbon are defined as parallel graphene sheets (Fig. 1, left). We explore adsorption in pores with widths $H=1,2,3$, and $4 \mathrm{~nm}$ ( $\mathrm{H}$ is defined as the distance in $z$ direction between the centers of the carbon atoms in the graphene walls). In the $x y$ plane, the pores are infinitely large (modeled using periodic boundary conditions). The SURMOF structure (Fig. 1, right) studied here is one of the largest MOF structures reported so far. It exhibits P4 symmetry, and, for the longest organic linker (pentaphenyldicarboxylic acid, PPDC) [16]), its channel size is $2.8 \times 2.8 \mathrm{~nm}^{2}$. In the calculations, the channels were modeled as infinitely long pores using periodic boundary conditions.

\section{Results}

\section{Adsorption mechanism and layering transition}

At $80 \mathrm{~K}$, the adsorption of methane layers in slit pores is very regular. This is a consequence of our choice of an adsorbent model where the slit walls have the same structure as regular graphene. Corrugation of the graphene surface is negligible compared to the mean graphene-methane interaction. The adsorption isotherm is stepped (Fig. 2a); each subsequent adsorbed layer is very stable and well ordered. However, when the current layer attains its maximal capacity, the increasing pressure destabilizes the adsorbed system before the new stable layer is formed [9]. The density distribution (Fig. 2d) shows well-defined and separated peaks, indicating that the adsorbed methane is highly localized in the layers, just as in a solid structure. The solid character of the adsorbed structure was confirmed by calculating the order parameter $\Phi_{6}(\cong 1)$.

Increasing the temperature destabilizes the layered structure. Both the isotherms and the mass distributions change continuously between $80 \mathrm{~K}$ and $180 \mathrm{~K}$ (the critical temperature of methane). The whole system evolves from a solid below $80 \mathrm{~K}$ to a liquid above $180 \mathrm{~K}$. However, the melting does not occur uniformly across the whole pore volume.
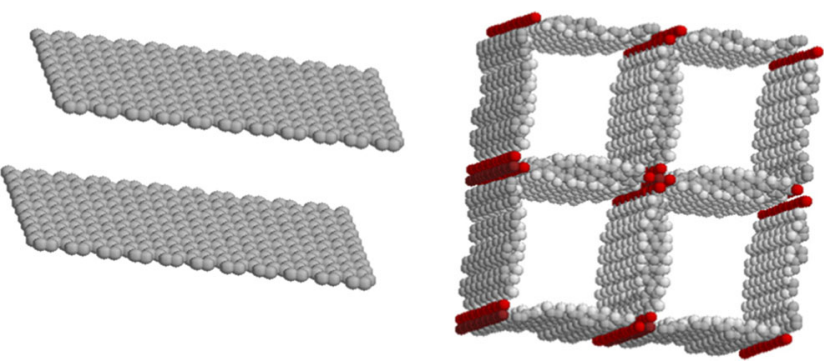

Fig. 1 Slit pore (left, graphene walls) and SURMOF (right) structures 
Calculations of the order parameters in each layer show that the layers melt at different temperatures [9]. It is important to emphasize that as the temperature is increased in the above range, the layering transitions also become more and more continuous (Fig. 2a-c). The system starts to melt from the middle part of the pore; the contact layers remain solid up to much higher temperatures, as can be seen from the density distributions (Fig. 2d-f); even above $140 \mathrm{~K}$, the contact layer is well defined and well separated from the internal filling of the pore (Fig. 2e,f). This proves that, even in the melted state, the system remains very heterogeneous and the molecules in the contact layer are more localized (in the $z$ direction) than those that are closer to the pore center.
The melting mechanism in the slit pores depends on the strength of attraction between the adsorbed molecules and the walls, as has already been reported [6, 7]. However, we emphasize here that there is a strong gradient in the strength of attractive forces between the adsorbed molecules and the walls. This means that the adsorbed system is heterogeneous, so the melting process is a continuous function of temperature. Figure 3 a shows the evolution of the average filling density of the pore. Only a very small jump in filling density at $\sim 90 \mathrm{~K}$ is observed for the slit pore. This discontinuity has a tendency to disappear when the modeled system is larger in the $x y$ direction. This result is observed for a specific thermodynamic path that is chosen for the phase transformation. In the adsorbed
Fig. 2 Methane adsorption in slit pores at $80 \mathrm{~K}(\mathbf{a}, \mathbf{d}), 140 \mathrm{~K}(\mathbf{b}, \mathbf{e})$, and $180 \mathrm{~K}(\mathbf{c}, \mathbf{f})$. a, b, c Adsorption isotherms in pores $(\mathrm{H}=1 \mathrm{~nm}, 2 \mathrm{~nm}, 3 \mathrm{~nm}$, and $4 \mathrm{~nm})$. d, e, f Density distributions in $\mathrm{H}=3 \mathrm{~nm}$ pore. There is a correlation between isotherm evolution and the melting of the methane inside the pore
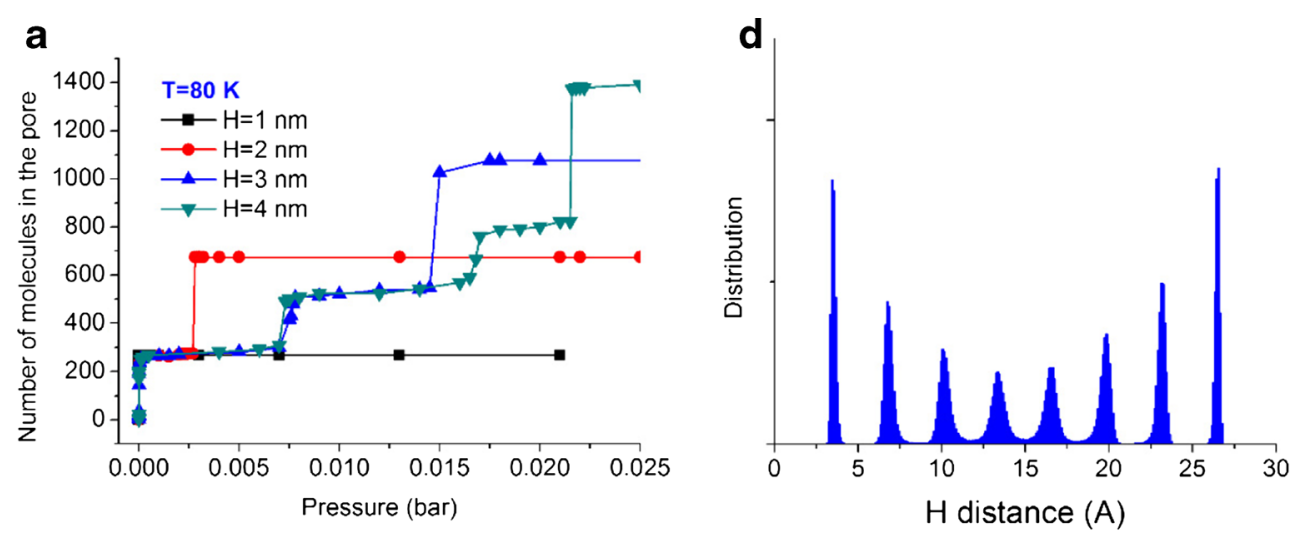

b
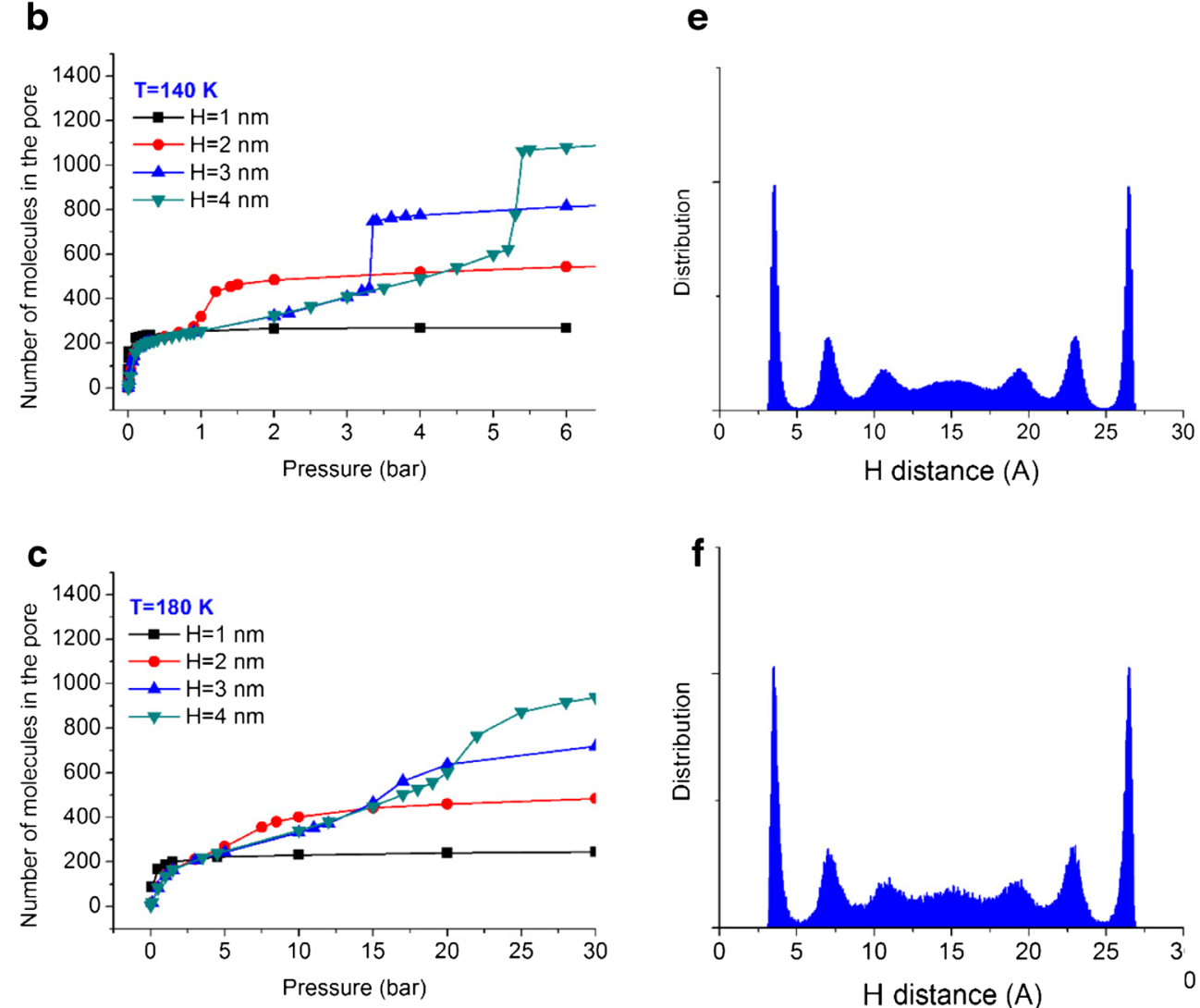

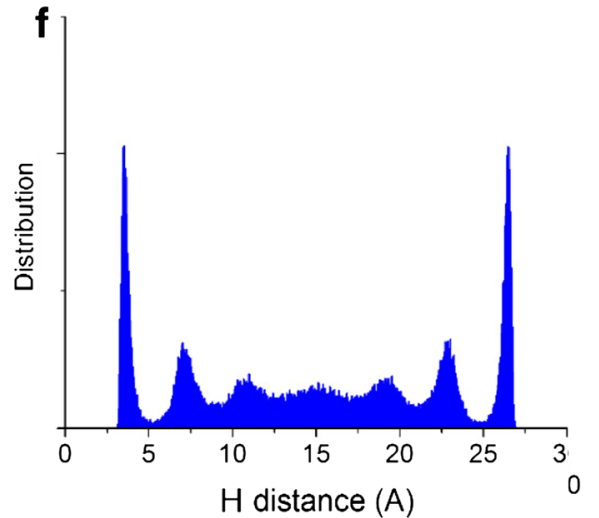


Fig. 3 Melting in pores. Density as a function of temperature in the 3-nm slit pore (a) and in SURMOF (b). The lines are only included for visual guidance a

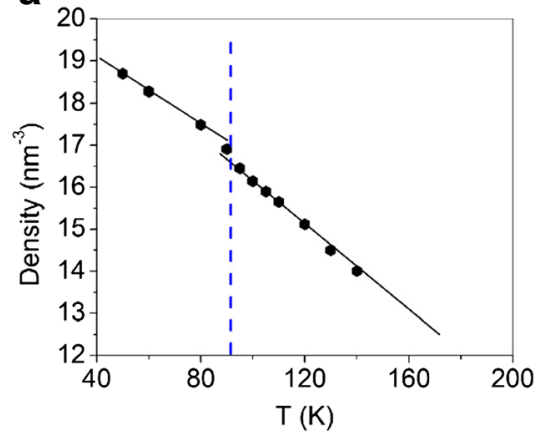

b

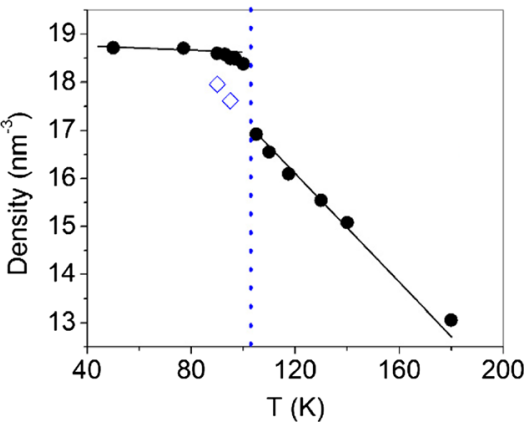

system, there are many possible choices of path that are defined by the pressure of the external gas. Any path between the bulk coexistence curve and the capillary condensation curve could be studied [1]. In this work, we adopted the path in the middle between those two limits. As the density of the system varies depending on the path, this choice may affect the observed mechanism of melting and make it more discontinuous.

\section{Melting mechanism in SURMOF pores}

The adsorption mechanism is strongly modified when the pore geometry changes from slit-like to channel-like with a square cross-section. Although the lateral dimensions of the square channels of the SURMOF studied here are comparable to the size of a 3-nm graphene-based slit pore, the energy landscape is totally different. The average energy of adsorption increases due to the cumulative contributions from all channel walls. This leads to rapid pore filling at pressures lower than in the slit pore. At $80 \mathrm{~K}$ and the pressure at which only the first and second layers are adsorbed in the slit pore, the SURMOF pore is totally filled. The influences of the pore geometry and stronger interactions with the walls are clearly observed in the density curve (Fig. 3b): the singularity at melting is much more pronounced, and a small hysteresis between melting and freezing is observed. The detailed evolution of the density has been discussed in a previous paper [9].

Below $100 \mathrm{~K}$, the methane adsorbed in SURMOF pores presents a solid structure. The order in the $x y$ plane is defined by the P4 tetragonal symmetry of the SURMOF (Fig. 4). At the same time, in the $x z$ and $y z$ planes, the adsorbed molecules show pseudohexagonal order; that is, the arrangement of molecules is not perfect due to the nanometric size of the $x y$ planes and noncommensurability between the adsorbent framework and the methane-methane equilibrium distance. This leads to minor deformations of the adsorbed structure that can be clearly seen in the density distribution shown in Fig. 4. Below the melting temperature (estimated to be $T_{\mathrm{m}}=102 \pm 2 \mathrm{~K}$ ), the average density distribution is nearly constant. This is a consequence of the ordered structure, which does not change until the methane is melted.
The melting transition is gradual: after the initial abrupt $10 \%$ decrease at $\sim 102 \mathrm{~K}$, the density of the adsorbed methane decreases continuously from 17 molecules $/ \mathrm{nm}^{3}$ to 13 molecules $/ \mathrm{nm}^{3}$ at $180 \mathrm{~K}$. This gradual melting arises from the nanosize of the pores in the $x y$ plane: the resulting strong interaction with the SURMOF framework defines the melting mechanism. The contact layers of methane remain localized by the framework's corrugation up to the highest simulated temperature $(T=180 \mathrm{~K})$. At the same time, the fluctuations of the molecules close to the channel's center increase with temperature (Fig. 5) because the density of adsorbed methane is lower at higher temperatures. Consequently, the melted structure is strongly heterogeneous: when the center of the channel is already liquid, the molecules in the contact layers stay in a solid-like state stabilized by interactions with the pore walls. This observation is confirmed by the results of a calculation of the distribution of the order parameter (Fig. 5a, b, f, g).

Calculating the distribution of the order parameter (calculated locally in the pore) reveals more intricate behavior. Figure 5 shows the structural details of the phase evolution of methane adsorbed in SURMOF between $90 \mathrm{~K}$ and $180 \mathrm{~K}$. We compare the distributions of $\Phi_{4}$ and $\Phi_{6}$, both of which were calculated locally, within the adsorbed layer. $\Phi_{4}$ represents the molecular order calculated in layers parallel to the $x y$ plane, whereas $\Phi_{6}$ is defined in layers parallel to the $x z$ and $y z$ planes. These parameters show similar evolutions with the melting process, presenting transition temperatures of between $100 \mathrm{~K}$ and $105 \mathrm{~K}$. The behavior of the parameters
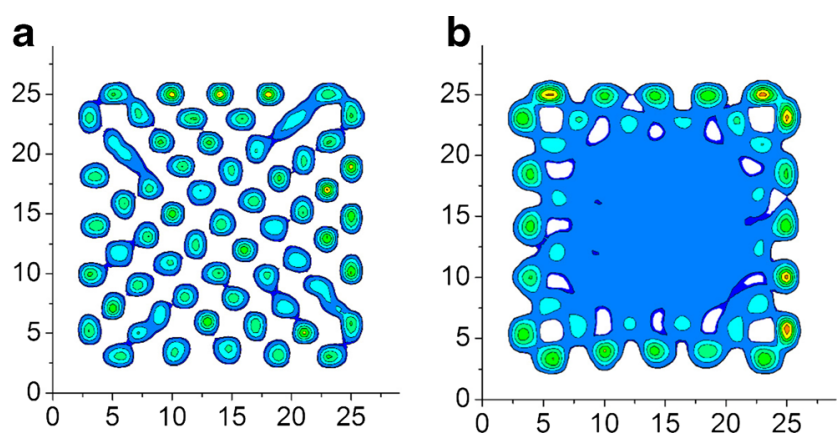

Fig. 4 Density distribution of methane molecules in a SURMOF pore before melting at $105 \mathrm{~K}$ (a) and at $180 \mathrm{~K}$ (b) 
Fig. 5 Melting in a SURMOF pore. Order parameter

distributions (a, b, c, d, e: $\Phi_{4} ; \mathbf{f}, \mathbf{g}$,

$\left.\mathbf{h}, \mathbf{i}, \mathbf{j}: \Phi_{6}\right)$ in a pore above the melting temperature $(\mathbf{a}, \mathbf{f}: 90 \mathrm{~K} ; \mathbf{b}$, g: $100 \mathrm{~K} ; \mathbf{c}, \mathbf{h}: 105 \mathrm{~K}$; d, i: $110 \mathrm{~K}$; $\mathbf{e}, \mathbf{j}: 180 \mathrm{~K})$. The color scale changes from 1 (red) to 0 (black); green indicates values of 0.45 0.55
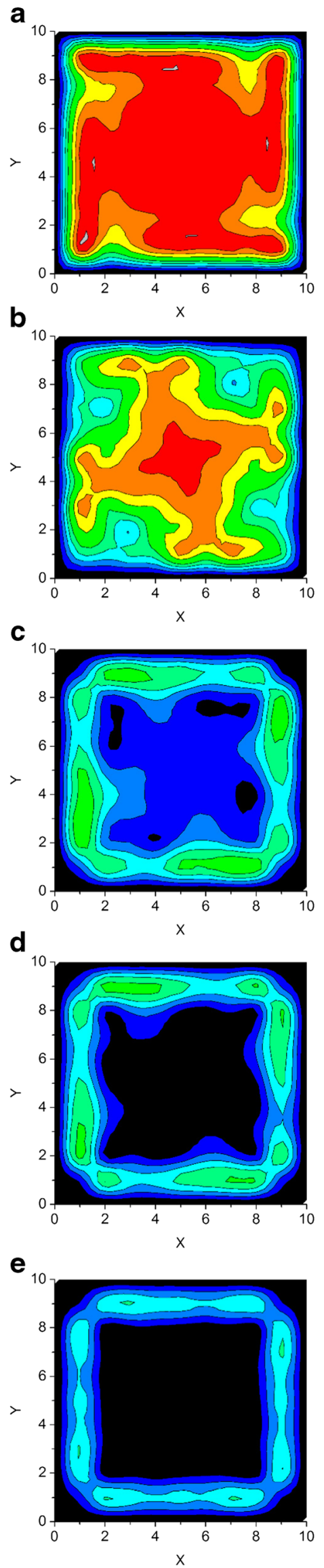

f

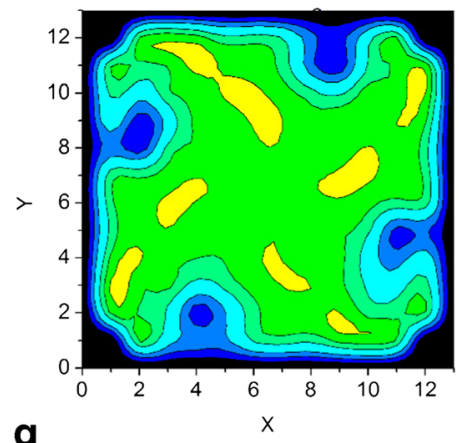

g

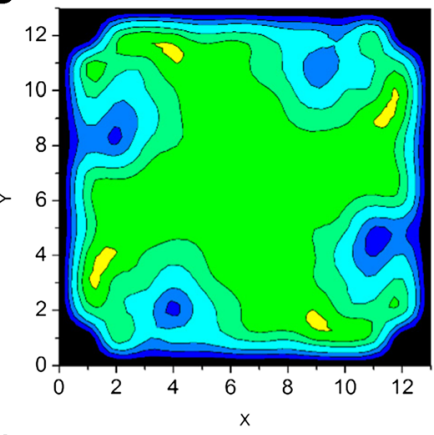

h

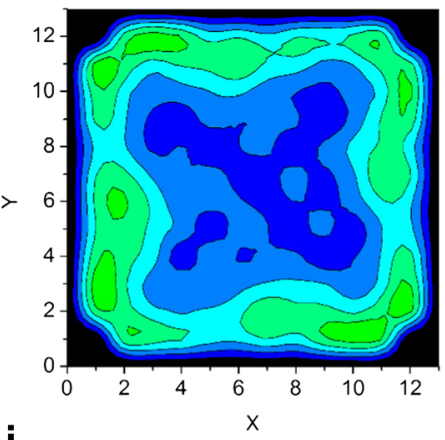

i

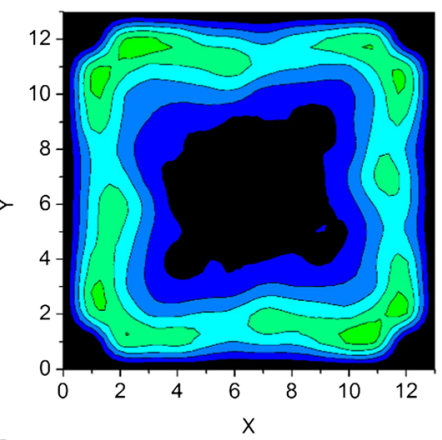

j

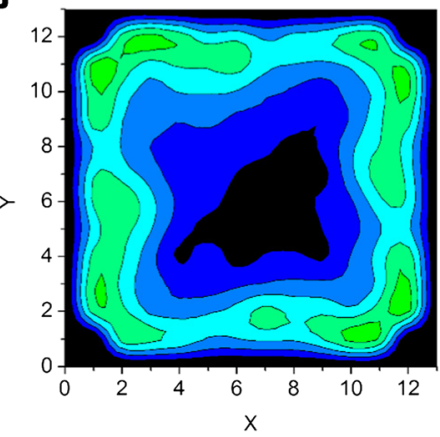



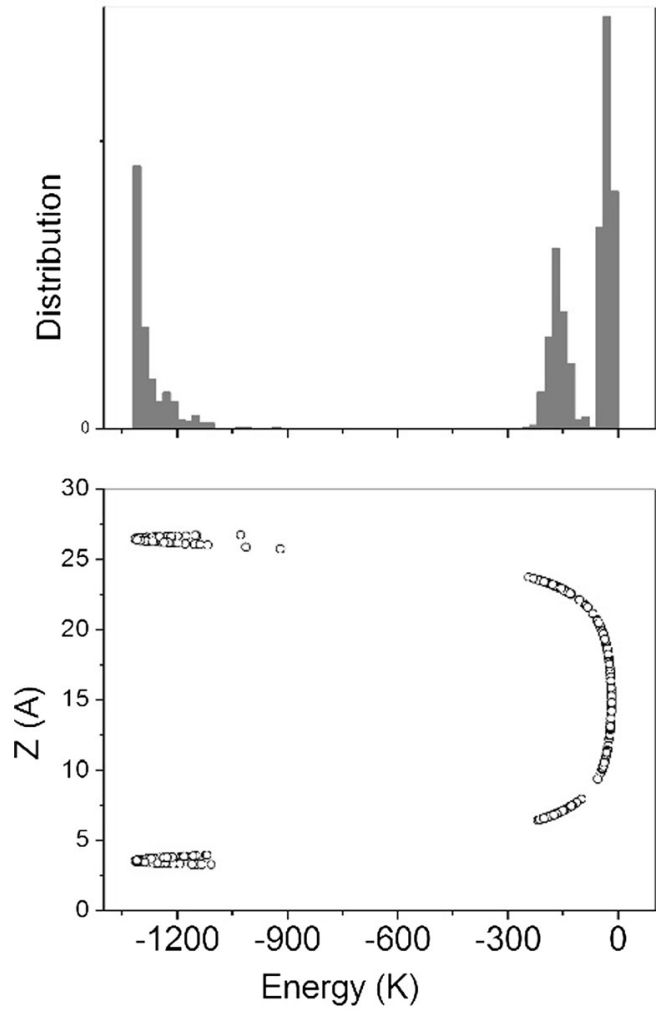

Fig. 6 Distribution of the wall-methane interaction energy (top) and its correlation with the $z$ positions of the molecules in the pore (bottom) at $80 \mathrm{~K}$

shows that (i) the contact layers are deformed at low temperature and (ii) the contact layers stay less dynamic than the interior of the channel at temperatures above the melting point. The hexagonal order detected in the $x z$ and $y z$ planes is much less perfect than the tetragonal order, although it exists across a much wider range of temperatures. At the highest temperatures, the tetragonal order totally disappears except in the contact layer, whereas the hexagonal order is still detectable in two contact layers.

\section{Conclusions}

The melting mechanism for fluids confined in nanospaces depends on the size and shape of the space and the properties of the confining environment. In nanopores $<3 \mathrm{~nm}$ in size, local characterization of the density and structure is necessary to explain unusual characteristics of the melting mechanism [17]. The important result of the present study is the observation that the contact layer behaves very differently from the interior of the pore. This is not surprising, because the contact layer is influenced the most by the interaction with the pore wall. However, when the pore size is $<5 \mathrm{~nm}$, the contact layer propeties affect the phase behavior throughout the pore. For larger pores, the influence of the contact layer is not as pronounced and for macroscopic pores totally negligible.
Our modeling results are coherent with existing experimental measurements. Direct observations of layer structure are possible using X-ray diffraction. The characteristic behavior of the contact layers was deduced experimentally from a $\mu-T$ (chemical potential vs. temperature) phase diagram for argon condensed in a porous glass matrix [18]. Electron microscopy has the appropriate spatial resolution to observe geometric features of liquids in nanopores, but it is an ultrahighvacuum technique, so applying the technique in this context is difficult (but not impossible [19]) due to the high vapor pressure of adsorbed liquids. The different dynamic properties of the contact layers with respect to those of the interior of the pore can be studied using incoherent quasi-elastic neutron scattering measurements [20].

Studies of the structural phase transformations in nanopores are computationally more demanding than those of their bulk analogs. This is mostly because these structures, which are composed of just a few molecular layers, are very heterogeneous. Figure 6 shows how heterogeneous the $3-\mathrm{nm}$ slit pore is at $80 \mathrm{~K}$. Therefore, the equilibrium includes the solid-state contact layer and liquid-state second layer. This equilibrium is easily disturbed by small fluctuations in the density of the adsorbed system. In addition, as the melting mechanism depends on the layer position and on the particular thermodynamic melting path chosen, the stabilization of the system under given thermodynamic conditions is a long process. In nanopores $<5 \mathrm{~nm}$ in size, there is another factor that influences the mechanism of transformation. The adsorbed phase structure can easily be incommensurate with the size of the pore. This introduces additional deformations of the contact layers that may locally destabilize the adsorbed phase. We observed this effect in the SURMOF pores. This effect leads to peculiar local deformations of the distribution of the order parameter (see Fig. 5), which still preserve the fourfold symmetry of the SURMOF unit cell.

It is important to emphasize that the melting transition is closely correlated with the adsorption mechanism: the evolution of the stepped adsorption isotherm into a more continuous one as the temperature is increased is an indication that the adsorbed system becomes more liquid.

The examples studied above clearly show that the melting transition exhibits intricate behavior when it occurs in nanopores. Contrary to the 3D bulk case, it is highly nonhomogeneous and may shift between discontinuous and continuous types. A full understanding of this phenomenon requires further fundamental studies.

Acknowledgements Supported by the Polish National Science Center (NCN, grant no. 2015/17/B/ST8/00099). The calculations were partially performed at the WCSS computer center of The Wroclaw University of Science and Technology (grant no. 33). 


\section{References}

1. Miyahara M, Gubbins KE (1997) Freezing/melting phenomena for Lennard-Jones methane in slit pores: a Monte Carlo study. J Chem Phys 106:2865. doi:10.1063/1.473415

2. Maddox MW, Gubbins KE (1997) A molecular simulation study of freezing/melting phenomena for Lennard-Jones methane in cylindrical nanoscale pores. J Chem Phys 107:9659-67. doi:10.1063 $/ 1.475261$

3. Radhakrishnan R, Gubbins KE (1999) Free energy studies of freezing in slit pores: an order parameter approach using Monte Carlo simulation. Mol Phys 96:1249-1267

4. Nguyen TX, Bhatia SK, Nicholson D (2002) Close packed transitions in slit-shaped pores: density functional theory study of methane adsorption capacity in carbon. J Chem Phys 117:10827-36

5. Ayappa KG, Ghatak C (2002) The structure of frozen phases in slit nanopores: a grand canonical Monte Carlo study. J Chem Phys 117: 5373-83. doi:10.1063/1.1501120

6. Radhakrishnan R, Gubbins KE, Sliwinska-Bartkowiak M (2002) Global phase diagrams for freezing in porous media. J Chem Phys 116:1148-56

7. Alba-Simionesco C, Coasne B, Dosseh G, Dudziak G, Gubbins KE, Radhakrishnan R, Sliwinska-Bartkowiak M (2006) Effects of confinement on freezing and melting. J Phys Condens Matter 18: R15-R68. doi:10.1088/0953-8984/18/6/R01

8. Christenson HK (2001) Confinement effects on freezing and melting. J Phys Condens Matter 13:R95-R133

9. Dundar E, Boulet P, Wexler C, Firlej L, Llewellyn P, Kuchta B (2016) Heterogeneous melting of methane confined in nano-pores. J Chem Phys 145:144704. doi:10.1063/1.4964650

10. Etters RD, Kuchta B, Belak J (1993) Vacancy induced melting of $\mathrm{N}_{2}$ adlayers on graphite. J Phys Rev Lett 70:826-829
11. Kuchta B, Luty T (1983) Lattice-dynamics of solid nitrogen with an abinitio intermolecular potential. 2. Anharmonic librations in the alpha-beta phase. J Chem Phys 78:1447-1452

12. Kuchta B, Firlej L, Pfeifer P, Wexler C (2010) Numerical estimation of physical limits of hydrogen storage by physisorption in microporous nanospaces. Carbon 48:223-231

13. Golebiowska M, Roth M, Firlej L, Kuchta B, Wexler C (2012) The reversibility of the adsorption of methane-methyl mercaptan mixtures in nanoporous carbon. Carbon 50:225-234. doi:10.1016/j. carbon.2011.08.039

14. Nicholson D, Parsonage G (1982) Computer simulation and the statistical mechanics of adsorption. Academic, London

15. Razak M, Do DD, Birkett GR (2011) Evaluation of the interaction potentials for methane adsorption on graphite and in graphitic slit pores. Adsorption 17:385-394. doi:10.1007/s10450-011-9335-5

16. Liu J, Lukose B, Shekhah O, et al. (2012) A novel series of isoreticular metal organic frameworks: realizing metastable structures by liquid phase epitaxy. Sci Rep 2:921. doi:10.1038 /srep00921

17. Malijevský A (2016) Phase transitions of fluids in heterogeneous pores. Condens Matter Phys 19:1-18

18. Huber P, Knorr K (1999) Adsorption-desorption isotherms and Xray diffraction of $\mathrm{Ar}$ condensed into a porous glass matrix. Phys Rev B 60:12657. doi:10.1103/PhysRevB.60.12657

19. Huber P (2015) Soft matter in hard confinement: phase transition thermodynamics, structure, texture, diffusion and flow in nanoporous media. J Phys Condens Matter 27:103102. doi:10.1088/0953-8984/27/10/103102

20. Hofmann T, Wallacher D, Mayorova M, Zorn R, Frick B, Huber P (2012) Molecular dynamics of $n$-hexane: a quasi-elastic neutron scattering study on the bulk and spatially nanochannel-confined liquid. J Chem Phys 136:124505. doi:10.1063/1.3696684 